\title{
Annotation of Genes Having Candidate Somatic Mutations in Acute Myeloid Leukemia with Whole-Exome Sequencing Using Concept Lattice Analysis
}

\author{
Kye Hwa Lee, Jae Hyeun Lim, Ju Han Kim \\ Division of Biomedical Informatics, Seoul National University Biomedical Informatics (SNUBI) and Systems Biomedical \\ Informatics National Core Research Center, Seoul National University College of Medicine, Seoul 110-799, Korea
}

http://dx.doi.org/10.5808/GI.2013.11.1.38

Genomics Inform 2013;11(1):38-45

The funding acknowledgment in this article was partially omitted as published. Additional acknowledgment is as follows:

This work was supported by the basic science research program through the National Research Foundation of Korea (NRF), funded by the Ministry of Education, Science and Technology (2012-0000994). This material is based upon work supported by a grant of the National Project for Personalized Genomic Medicine, Ministry for Health \& Welfare, Republic of Korea (A111218). 